\title{
Propagation in Constraints: How One Thing Leads to Another
}

\author{
Ian P. Gent \\ School of Computer Science, University of St. Andrews, \\ St. Andrews, Scotland, UK \\ ipg@cs.st-andrews.ac.uk
}

\section{Abstract of Invited Talk}

At a conference such as CPAIOR, we have experts from many different approaches to searching huge combinatorial spaces. Much of what we all do is common, for example similar search methods, heuristics, and learning techniques. So what is it that is essentially different about Constraint Programming in particular? One answer is the power and diversity of constraint propagation algorithms. By contrast, other search disciplines often rely on just one propagation technique, such as unit propagation in SAT.

So to paraphrase the property expert, for the duration of this talk I'll say that the three most important aspects of Constraint Programming are "Propagation, Propagation, Propagation." I'll talk about all three. First, what Propagation is and the theory of how propagation algorithms work. Second, how Propagation forms the beating heart of a modern constraint solver, typically throwing away a lot of the theory in the process. And third, some aspects of Propagation in constraints being researched right now.

I hope to bring out some of the beauty and surprises in propagation. I also hope to show how ideas from one discipline have propagated to another, for example the introduction of watched literals from SAT into Constraint Programming. I also hope one propagation will lead to another as some of these lovely ideas propagate again. 